\title{
Analyses of Inverse Kinematics, Statics and Workspace of a Novel 3RPS- 3SPR Serial-Parallel Manipulator
}

\author{
Bo $\mathrm{Hu}^{*}, 1$, Yi Lu${ }^{1}$, Jing Jing $\mathrm{Yu}^{2}$ and Shan Zhuang ${ }^{1}$ \\ ${ }^{I}$ Robotics Research Center, College of Mechanical Engineering, Yanshan University, Qinhuangdao, Hebei \\ Qinhuangdao, China \\ ${ }^{2}$ Department of Mechanics and Electronics, Heibei Normal University of Science \& Technology, Hebei Qinhuangdao, \\ China
}

\begin{abstract}
A novel 3RPS-3SPR serial-parallel manipulator (S-PM) with 6 degree of freedoms (DOFs) is proposed in this paper. It includes a lower 3RPS parallel manipulator (PM) and an upper 3SPR PM. Its inverse kinematics, active forces and workspace are solved. First, the inverse displacement is solved in close form based on the geometrical and the dimensional constraints of this S-PM. Second, the $9 \times 9$ and $6 \times 6$ form inverse Jacobian matrices are derived and the active forces are solved using principle of virtual work. Third, the workspace of this S-PM is constructed by using CAD variation geometry approach.
\end{abstract}

Keywords: Serial-parallel manipulator, kinematics, statics, workspace.

\section{INTRODUCTION}

Recently, parallel manipulators (PMs) have attracted much attention due to their merits and industrial applications [1]. This kind of manipulators has been studied widely [2-7]. However, few investigations have been developed on SPMs.

The S-PM has merits of both serial manipulator (SM) and PM. This kind of manipulators is characterized by its high stiffness compared with SM and large workspace compared with PM. In this aspect, Tanev [8] solved the forward and inverse position problems of a hybrid manipulator. $\mathrm{Lu}$ and $\mathrm{Hu}$ [9] solved active forces of a 2(3-SPR) S-PM by CAD variation geometry approach, and solved the forward kinematics of this S-PM [10] based on the analytical results of 3RPS PM. Jaime[11] studied the kinematics and dynamics of 2(3-RPS) manipulators by means of screw theory and the principle of virtual work. Zheng et al., [12] studied an S-PM which possess of a pure translational and a pure rotational 3UPU PMs. O. Ibrahim and W. Khalil [13] proposed a method for the calculation of the inverse and direct dynamic models for S-PMs using recursive Newton Euler formalism. A. Ramadan [14] proposed a compact but yet economical two-fingered micro-nano hybrid manipulator hand. Liu [15] studied an S-PM formed by adding a 2-dof mechanism on Tricept.

In Gallardo's work [11], an improved 2(3SPR) S-PM with a more compact topology than the original one proposed by $\mathrm{Lu}$ [9] was investigated. The improved 2(3SPR) SPM with compact topology can effectively diminish undesirable deflections and the presence of bending moments over

*Address correspondence to this author at the Robotics Research Center, College of Mechanical Engineering, Yanshan University, Qinhuangdao, Hebei Qinhuangdao, China; Tel: 8613230307616; Fax: 86-335-8074783; E-mail: hbz0001@yahoo.com.cn the kinematical pairs which affecting the accuracy of this manipulator [11]. From this point of view, a novel S-PM which consists of a 3RPS PM [16-18] and its invertible structure 3SPR PM [19] adopting compact topology by using compound spherical joint [20] is investigated in this paper.

The forward kinematics of this kind of manipulators has been studied [8-12]. However, the inverse kinematics of this kind of manipulators is a difficult work and hasn't been attempted. Inverse kinematics is a common issue for kinematics analysis and plays an important role in control. This paper focuses on establishing the inverse displacement, inverse velocity and active forces of a 3 RPS-3SPR S-PM based on the dimension and geometrical constraints. It is also a challenging work to solve the workspace of 3RPS-3SPR SPM due to its complicated kinematics. The workspace of this $\mathrm{S}-\mathrm{PM}$ is solved using CAD variation geometry approach in this paper.

\section{CHARACTERISTICS OF 3RPS-3SPR S-PM}

The 3RPS-3SPR S-PM with 6-DOF is consisted of a lower 3RPS PM and an upper 3SPR PM. The 3RPS and 3SPR PMs are connected serially. The lower 3RPS PM includes a moving platform $m_{b}$, a base $B$, and three extendable active limbs $r_{b \mathrm{i}}(i=1,2,3)$ with their linear actuators. The upper 3SPR PM includes a moving platform $m$, a base $m_{b}$ which is simultaneously used as the moving platform of the lower PM, and three extendable driving limbs $r_{c \mathrm{i}}(i=1,2,3)$ with their linear actuators. $m$ is a equilateral triangle $\Delta c_{1} c_{2} c_{3}$ with $o$ as its center and $l_{i}=l$ as its side. $B$ is an equilateral triangle $\Delta a_{1} a_{2} a_{3}$ with $O$ as its center and $L_{i}=L$ as its side. $m_{b}$ is a equilateral triangle $\Delta b_{1} b_{2} b_{3}$ with $d_{i}=d$ as its sides.

Three identical RPS limbs $r_{b \mathrm{i}}(i=1,2,3)$ of the lower 3RPS PM connect $m_{b}$ to $B$ by a spherical joint $\mathrm{S}$ on $m_{b}$ at $b_{i}$, a driving limb with a prismatic joint $P$, and a revolute joint $R$ 
on $B$ at $a_{i}$, respectively. The $R$ joint on $B$ at $a_{i}$ is parallel with the subtense of $a_{i}$. Three identical SPR limbs $r_{c i}(i=1,2,3)$ of the upper 3SPR PM connect $m$ to $m_{b}$ by a revolute joint $R$ on $m$ at point $c_{i}$, a driving limb with a prismatic joint $P$, and a spherical joint $S$ on $m_{b}$ at $b_{i}$, respectively. The $R$ joint on $m$ at $c_{i}$ is parallel with the subtense of $c_{i}$. Let $\{B\}$ be a coordinate $O-X Y Z$ with $O$ as its origin fixed on $B$ at $O,\{m\}$ be a coordinate $o-x y z$ with $o$ as its origin fixed on $m$ at $o$. Let $\|$ and $\perp$ be parallel and perpendicular constraints, respectively. Some constraints $\left(X\left\|a_{1} a_{3}, Y \perp a_{1} a_{3}, Z \perp B, x\right\| c_{1} c_{3}, y \perp c_{1} c_{3}, z \perp m\right)$ are satisfied, see Fig. (1).

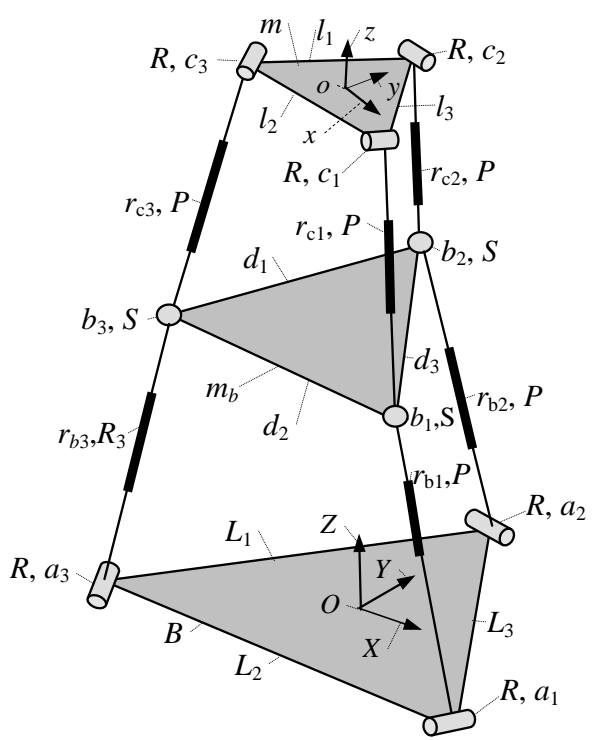

Fig. (1). 3RPS-3SPR S-PM.

\section{INVERSE DISPLACEMENT ANALYSIS}

The position vectors $\boldsymbol{a}_{i}(i=1,2,3)$ of vertices $a_{i}$ in $\{B\}$ can be expressed as follows

$$
\begin{aligned}
& \boldsymbol{a}_{1}=\left[\begin{array}{l}
a_{1 x} \\
a_{1 y} \\
a_{1 z}
\end{array}\right]=\left[\begin{array}{c}
q E / 2 \\
-E / 2 \\
0
\end{array}\right], \boldsymbol{a}_{2}=\left[\begin{array}{c}
a_{2 x} \\
a_{2 y} \\
a_{2 z}
\end{array}\right]=\left[\begin{array}{l}
0 \\
E \\
0
\end{array}\right], \\
& \boldsymbol{a}_{3}=\left[\begin{array}{c}
a_{3 x} \\
a_{3 y} \\
a_{3 z}
\end{array}\right]=\left[\begin{array}{c}
-q E / 2 \\
-E / 2 \\
0
\end{array}\right]
\end{aligned}
$$

The position vectors $\boldsymbol{c}_{i}(i=1,2,3)$ of vertices $c_{i}$ in $\{m\}$ can be expressed as follows

$$
{ }^{m} \boldsymbol{c}_{1}=\left[\begin{array}{c}
q C / 2 \\
-C / 2 \\
0
\end{array}\right],{ }^{m} \boldsymbol{c}_{2}=\left[\begin{array}{l}
0 \\
C \\
0
\end{array}\right],{ }^{m} \boldsymbol{c}_{3}=\left[\begin{array}{c}
-q C / 2 \\
-C / 2 \\
0
\end{array}\right]
$$

The position vectors $c_{i}$ of vertices $c_{i}$ in $\{B\}$ can be expressed as follows

$\boldsymbol{c}_{i}=\left[\begin{array}{c}c_{i x} \\ c_{i y} \\ c_{i z}\end{array}\right]=\mathrm{R}^{m} \boldsymbol{c}_{i}+\boldsymbol{o}$

$$
\begin{aligned}
& \boldsymbol{o}=\left[\begin{array}{lll}
X_{o} & Y_{o} & Z_{o}
\end{array}\right]^{T}, \mathrm{R}=\left[\begin{array}{lll}
\mathbf{x} & \mathbf{y} & \mathbf{z}
\end{array}\right] \\
& \boldsymbol{x}=\left[\begin{array}{lll}
x_{l} & x_{m} & x_{n}
\end{array}\right]^{T}, \boldsymbol{y}=\left[\begin{array}{lll}
y_{l} & y_{m} & y_{n}
\end{array}\right]^{T}, \boldsymbol{z}=\left[\begin{array}{lll}
z_{l} & z_{m} & z_{n}
\end{array}\right]^{T}
\end{aligned}
$$

Here $X_{o}, Y_{o}$ and $Z_{o}$ are three position coordinates of $o, \mathbf{R}$ is a rotation transformation matrix from $\{B\}$ to $\{m\}, E$ denotes the distance from point $O$ to $a_{i}, C$ denotes the distance from point $o$ to $c_{i}$ and $q=3^{1 / 2}$.

Let $\alpha, \beta$ and $\lambda$ be three Euler angles. Let $\varphi$ be one of $(\alpha, \beta$, $\lambda), s_{\varphi}=\sin \varphi, c_{\varphi}=\cos \varphi$. Using $X-Y-X$ type Euler rotations, the rotation transformation matrix can be expressed as follows [16]:

$$
\mathbf{R}=\left[\begin{array}{ccc}
c_{\beta} & s_{\beta} s_{\lambda} & s_{\beta} c_{\lambda} \\
s_{\alpha} s_{\beta} & c_{\alpha} c_{\lambda}-s_{\alpha} c_{\beta} s_{\lambda} & -c_{\alpha} s_{\lambda}-s_{\alpha} c_{\beta} c_{\lambda} \\
-c_{\alpha} s_{\beta} & s_{\alpha} c_{\lambda}+c_{\alpha} c_{\beta} s_{\lambda} & -s_{\alpha} s_{\lambda}+c_{\alpha} c_{\beta} c_{\lambda}
\end{array}\right]
$$

Let $R_{i 1}(i=1,2,3)$ be the revolute joints at $a_{i}(i=1,2,3)$ on the lower PM, $R_{i 2}(i=1,2,3)$ be the revolute joints at $c_{\mathrm{i}}$ $(i=1,2,3)$ on the upper PM. Then the unit vectors $\boldsymbol{R}_{i j}$ of $R_{i j}$ $(i=1,2 ; j=1,2,3)$ in $\{B\}$ can be expressed as following

$$
\begin{aligned}
& \mathbf{R}_{11}=\frac{1}{2}\left[\begin{array}{lll}
1 & q & 0
\end{array}\right]^{T}, \mathbf{R}_{12}=\left[\begin{array}{lll}
1 & 0 & 0
\end{array}\right]^{T}, \mathbf{R}_{13}=\frac{1}{2}\left[\begin{array}{lll}
-1 & q & 0
\end{array}\right]^{T} \\
& \mathbf{R}_{21}=\mathrm{R} \mathbf{R}_{11}=\frac{1}{2}\left[\begin{array}{c}
x_{l}+q y_{l} \\
x_{m}+q y_{m} \\
x_{n}+q y_{n}
\end{array}\right], \mathbf{R}_{22}=\mathrm{R} \mathbf{R}_{21}=\left[\begin{array}{c}
x_{l} \\
x_{m} \\
x_{n}
\end{array}\right],
\end{aligned}
$$

$\mathbf{R}_{23}=\mathrm{R} \mathbf{R}_{31}=\frac{1}{2}\left[\begin{array}{c}-x_{l}+q y_{l} \\ -x_{m}+q y_{m} \\ -x_{n}+q y_{n}\end{array}\right]$ satisfy

In the lower and upper PMs, the geometrical constraints

$R_{i 1} \perp r_{b i}, R_{i 2} \perp r_{c i}(i=1,2,3)$

From Eq. (5a), it leads to

$\mathbf{R}_{i 1} \cdot\left(\boldsymbol{b}_{i}-\boldsymbol{a}_{i}\right)=0$

$\mathbf{R}_{i 2} \cdot\left(\boldsymbol{b}_{i}-\boldsymbol{c}_{i}\right)=0$

here, $\boldsymbol{b}_{i}$ denotes the position vectors of point $b_{i}(i=1,2,3)$.

Let $h_{i x}, h_{i y}, h_{i z}$ be three position coordinates of point $h_{i}(i=1,2,3)$, where $h$ comes form $a, b, c$.

From Eq. (5b), it leads to

$\left[\begin{array}{lll}1 & q & 0\end{array}\right]\left[\begin{array}{lll}b_{1 x}-a_{1 x} & b_{1 y}-a_{1 y} & b_{1 z}-a_{1 z}\end{array}\right]^{T}=0$

$\left[\begin{array}{lll}1 & 0 & 0\end{array}\right]\left[\begin{array}{lll}b_{2 x}-a_{2 x} & b_{2 y}-a_{2 y} & b_{2 z}-a_{2 z}\end{array}\right]^{T}=0$

$\left[\begin{array}{lll}-1 & q & 0\end{array}\right]\left[\begin{array}{lll}b_{3 x}-a_{3 x} & b_{3 y}-a_{3 y} & b_{3 z}-a_{3 z}\end{array}\right]^{T}=0$

From Eqs. (6a) to ( $6 \mathrm{c}$ ), it leads to

$$
\begin{aligned}
& b_{1 x}+q b_{1 y}=0 \Rightarrow b_{1 x}=-q b_{1 y} \\
& b_{2 x}=a_{2 x}=0 \\
& b_{3 x}-q b_{3 y}=0 \Rightarrow b_{3 x}=q b_{3 y}
\end{aligned}
$$


From (5c), it leads to

$\left[\begin{array}{lll}x_{l}+q y_{l} & x_{m}+q y_{m} & x_{n}+q y_{n}\end{array}\right]\left[\begin{array}{lll}b_{1 x}-c_{1 x} & b_{1 y}-c_{1 y} & b_{1 z}-c_{1 z}\end{array}\right]^{T}=0$

$\left[\begin{array}{lll}x_{l} & x_{m} & x_{n}\end{array}\right]\left[\begin{array}{lll}b_{2 x}-c_{2 x} & b_{2 y}-c_{2 y} & b_{2 z}-c_{2 z}\end{array}\right]^{T}=0$

$\left[\begin{array}{lll}-x_{l}+q y_{l} & -x_{m}+q y_{m} & -x_{n}+q y_{n}\end{array}\right]\left[\begin{array}{lll}b_{3 x}-c_{3 x} & b_{3 y}-c_{3 y} & b_{3 z}-c_{3 z}\end{array}\right]^{T}=0$

Expanding Eqs. (8a-c), it leads to

$-q\left(x_{l}+q y_{l}\right) b_{1 y}+\left(x_{m}+q y_{m}\right) b_{1 y}+\left(x_{n}+q y_{n}\right) b_{1 z}$

$=\left(x_{l}+q y_{l}\right) c_{1 x}+\left(x_{m}+q y_{m}\right) c_{1 y}+\left(x_{n}+q y_{n}\right) c_{1 z}$

$x_{m} b_{2 y}+x_{n} b_{2 z}=x_{l} c_{2 x}+x_{m} c_{2 y}+x_{n} c_{2 z}$

$q\left(-x_{l}+q y_{l}\right) b_{3 y}+\left(-x_{m}+q y_{m}\right) b_{3 y}+\left(-x_{n}+q y_{n}\right) b_{3 z}$

$=\left(-x_{l}+q y_{l}\right) c_{3 x}+\left(-x_{m}+q y_{m}\right) c_{3 y}+\left(-x_{n}+q y_{n}\right) c_{3 z}$

From Eqs. (9a-c), $b_{1 \mathrm{z}}, b_{2 \mathrm{z}}$ and $b_{3 \mathrm{z}}$ can be expressed as follows:

$$
\begin{gathered}
b_{1 z}=s_{11}+s_{12} b_{1 y}, \\
s_{11}=\frac{\left(x_{l}+q y_{l}\right) c_{1 x}+\left(x_{m}+q y_{m}\right) c_{1 y}+\left(x_{n}+q y_{n}\right) c_{1 z}}{x_{n}+q y_{n}}, \\
s_{12}=\frac{q x_{l}+3 y_{l}-x_{m}-q y_{m}}{x_{n}+q y_{n}}, \\
s_{21}=\frac{x_{l} c_{2 x}+x_{m} c_{2 y}+x_{n} c_{2 z}}{b_{n}}, s_{22}=\frac{-x_{m}}{x_{n}} \\
s_{31}=\frac{\left(-x_{l}+q y_{l}\right) c_{3 x}-\left(x_{m}-q y_{m}\right) c_{3 y}-\left(x_{n}-q y_{n}\right) c_{3 z}}{-x_{n}+q y_{n}} \\
b_{32}=s_{31}+s_{32} b_{3 y} \\
-x_{n}+q y_{n}
\end{gathered}
$$

From Eqs. $(7 \mathrm{a}-\mathrm{c})$ and Eqs. $(10 \mathrm{a}-\mathrm{c})$, the position vectors $\boldsymbol{b}_{i}$ $(i=1,2,3)$ of vertices $b_{i}$ can be expressed as follows:

$\mathbf{b}_{1}=\left[\begin{array}{c}-q b_{1 y} \\ b_{1 y} \\ s_{11}+s_{12} b_{1 y}\end{array}\right], \mathbf{b}_{2}=\left[\begin{array}{c}0 \\ b_{2 y} \\ s_{21}+s_{22} b_{2 y}\end{array}\right], \mathbf{b}_{3}=\left[\begin{array}{c}q b_{3 y} \\ b_{3 y} \\ s_{31}+s_{32} b_{3 y}\end{array}\right]$

From the dimension constraints of the $m_{b}$, it leads to

$\left(b_{1 x}-b_{2 x}\right)^{2}+\left(b_{1 y}-b_{2 y}\right)^{2}+\left(b_{1 z}-b_{2 z}\right)^{2}=d^{2}$

$\left(b_{3 x}-b_{2 x}\right)^{2}+\left(b_{3 y}-b_{2 y}\right)^{2}+\left(b_{3 z}-b_{2 z}\right)^{2}=d^{2}$

$\left(b_{1 x}-b_{3 x}\right)^{2}+\left(b_{1 y}-b_{3 y}\right)^{2}+\left(b_{1 z}-b_{3 z}\right)^{2}=d^{2}$

By substituting Eq. (11) into (12a-b), it leads to

$$
\begin{aligned}
& p_{15} b_{1 y}^{2}+p_{14} b_{2 y}^{2}+p_{13} b_{1 y} b_{2 y}+p_{12} b_{2 y}+p_{11} b_{1 y}+p_{10}=0 \\
& p_{25} b_{3 y}^{2}+p_{24} b_{2 y}^{2}+p_{23} b_{2 y} b_{3 y}+p_{22} b_{3 y}+p_{21} b_{2 y}+p_{20}=0 \\
& p_{35} b_{3 y}^{2}+p_{34} b_{1 y}^{2}+p_{33} b_{1 y} b_{3 y}+p_{32} b_{3 y}+p_{31} b_{1 y}+p_{30}=0
\end{aligned}
$$

Where $p_{15}=4+s_{12}^{2}, p_{14}=1+s_{22}^{2}, p_{13}=-2-2 s_{12} s_{22}$,

$p_{12}=-2 s_{22}\left(s_{11}-s_{21}\right), p_{11}=2 s_{12}\left(s_{11}-s_{21}\right), p_{10}=\left(s_{11}-s_{21}\right)^{2}-d^{2}$,

$p_{25}=4+s_{32}^{2}, p_{24}=1+s_{22}^{2}, p_{23}=-2-2 s_{32} s_{22}, p_{22}=2 s_{32}\left(s_{31}-s_{21}\right)$,

$p_{21}=-2 s_{22}\left(s_{31}-s_{21}\right), p_{20}=\left(s_{31}-s_{21}\right)^{2}-d^{2}$,

$p_{35}=4+s_{32}^{2}, p_{34}=4+s_{12}^{2}, p_{33}=4-2 s_{32} s_{12}, p_{32}=2 s_{32}\left(s_{31}-s_{11}\right)$,

$p_{31}=-2 s_{12}\left(s_{31}-s_{11}\right), p_{30}=\left(s_{31}-s_{11}\right)^{2}-d^{2}$,

From Eqs. (13), it leads to

$$
\begin{aligned}
& k_{12} b_{1 y}^{2}+k_{11} b_{1 y}+k_{10}=0 \\
& k_{22} b_{3 y}^{2}+k_{21} b_{3 y}+k_{20}=0 \\
& k_{32} b_{3 y}^{2}+k_{31} b_{3 y}+k_{30}=0
\end{aligned}
$$

here

$$
\begin{aligned}
& k_{12}=p_{15}, k_{11}=p_{11}+p_{13} b_{2 y}, \\
& k_{10}=p_{14} b_{2 y}^{2}+p_{12} b_{2 y}+p_{10} \\
& k_{22}=p_{25}, k_{21}=p_{23} b_{2 y}+p_{22}, \\
& k_{20}=p_{24} b_{2 y}^{2}+p_{21} b_{2 y}+p_{20} \\
& k_{32}=p_{35}, k_{31}=p_{33} b_{1 y}+p_{32}, \\
& k_{30}=p_{34} b_{1 y}^{2}+p_{31} b_{1 y}+p_{30}
\end{aligned}
$$

From Eq. (14a), it leads to

$b_{3 y}^{2}=\frac{k_{21} k_{30}-k_{31} k_{20}}{k_{31} k_{22}-k_{21} k_{32}}$

From Eq. (14b), it leads to

$b_{3 y}=\frac{k_{22} k_{30}-k_{32} k_{20}}{k_{32} k_{21}-k_{22} k_{31}}$

From Eqs. (15a-b), it leads to

$\frac{k_{21} k_{30}-k_{31} k_{20}}{k_{31} k_{22}-k_{21} k_{32}}=\left(\frac{k_{22} k_{30}-k_{32} k_{20}}{k_{32} k_{21}-k_{22} k_{31}}\right)^{2}$

From Eq. (16), it leads to

$$
t_{14} b_{1 y}^{4}+t_{13} b_{1 y}^{3}+t_{12} b_{1 y}^{2}+t_{11} b_{1 y}+t_{10}=0
$$

here

$$
\begin{aligned}
& t_{14}=k_{22}^{2} p_{34}^{2}, t_{13}=2 k_{22}^{2} p_{34} p_{31}+k_{21} k_{22} p_{33} p_{34}, \\
& t_{12}=k_{22}^{2}\left(p_{31}^{2}+2 p_{30} p_{34}\right)-p_{34}\left(2 k_{20} k_{22} k_{32}+k_{21}^{2} k_{32}\right)+ \\
& k_{21} k_{22}\left(p_{33} p_{31}+p_{32} p_{34}\right)-k_{20} k_{22} p_{33}^{2}, \\
& t_{11}=2 k_{22}^{2} p_{30} p_{31}-p_{31}\left(2 k_{20} k_{22} k_{32}+k_{21}^{2} k_{32}\right)+ \\
& k_{21} k_{22}\left(p_{33} p_{30}+p_{32} p_{31}\right)-2 k_{20} k_{22} p_{32} p_{33}+k_{20} k_{21} k_{32} p_{33}, \\
& t_{10}=k_{22}^{2} p_{30}^{2}-p_{30}\left(2 k_{20} k_{22} k_{32}+k_{21}^{2} k_{32}\right)+k_{21} k_{22} p_{32} p_{30}- \\
& k_{20} k_{22} p_{32}^{2}+k_{20} k_{21} k_{32} p_{32}+k_{20}^{2} k_{32}^{2},
\end{aligned}
$$

From Eqs. (14a and 17), it leads to 


$$
\mathrm{U}\left[\begin{array}{c}
b_{1 y}^{5} \\
b_{1 y}^{4} \\
b_{1 y}^{3} \\
b_{1 y}^{2} \\
b_{1 y}^{2} \\
1
\end{array}\right]=0, \mathrm{U}=\left[\begin{array}{cccccc}
0 & t_{14} & t_{13} & t_{12} & t_{11} & t_{10} \\
t_{14} & t_{13} & t_{12} & t_{11} & t_{10} & 0 \\
0 & 0 & 0 & k_{12} & k_{11} & k_{10} \\
0 & 0 & k_{12} & k_{11} & k_{10} & 0 \\
0 & k_{12} & k_{11} & k_{10} & 0 & 0 \\
k_{12} & k_{11} & k_{10} & 0 & 0 & 0
\end{array}\right]
$$

The necessary condition for Eq. (18) to have nontrivial solutions is

$|\mathrm{U}|=0$

Eq. (19) is a polynomial about $b_{2 y}$. When $X_{o}, Y_{o}, Z_{o}, \alpha, \beta$ and $\lambda$ are given, $b_{2 y}$ can be solved from Eq. (19), $b_{1 y}$ and $b_{3 y}$ can be solved from Eq. (14a-b) and the coordinates $b_{i}(i=1,2$, 3) can be solved form Eq. (11). Then the inverse kinematics can be solved subsequently by the following equations.

$r_{b i}=\left|\boldsymbol{a}_{i}-\boldsymbol{b}_{i}\right|(i=1,2,3)$

$r_{c i}=\left|\boldsymbol{b}_{i}-\boldsymbol{c}_{i}\right|(i=1,2,3)$

\section{INVERSE JACOBIAN ANALYSIS}

The loop equation of $O a_{i} b_{i} c_{i} o(i=1,2,3)$ can be written as follows

$\boldsymbol{O a _ { i }}+\boldsymbol{a}_{i} \boldsymbol{b}_{i}+\boldsymbol{b}_{i} \boldsymbol{c}_{i}=\boldsymbol{O o}+\boldsymbol{o c} c_{i}$

By differentiating both sides of Eq. (21) with respect to time, it leads to

$v_{r b i} \delta_{b i}+\omega_{r b i} \times \boldsymbol{r}_{b i}+v_{r c i} \delta_{c i}+\omega_{r c i} \times \boldsymbol{r}_{c i}=\boldsymbol{v}+\omega \times \boldsymbol{e}_{i}$

Where $\boldsymbol{r}_{b i}$ and $\boldsymbol{r}_{c i}$ are the vectors, $\boldsymbol{\delta}_{b i}$ and $\boldsymbol{\delta}_{c i}$ are the unit vectors of $r_{b i}$ and $r_{c i}$, respectively. $v_{r b i}$ and $v_{r c i}(i=1,2,3)$ are the velocities, $\boldsymbol{\omega}_{r b i}$ and $\boldsymbol{\omega}_{r c i}$ are the angular velocity vectors of $r_{b i}$ and $r_{c i}$, respectively. $\boldsymbol{v}$ denotes the velocity of point $o, \omega$ denotes the angular velocity of $m$ and $\boldsymbol{e}_{i}$ denotes the vectors form $\boldsymbol{o}$ to $c_{i}$.

Based on the geometrical constraints of the upper manipulator, it leads to

$\boldsymbol{r}_{c i} \cdot \boldsymbol{R}_{i 2}=0$

Since $r_{b i}(i=1,2,3)$ rotate with $R_{i 1}(i=1,2,3)$, it leads to

$\omega_{r b i}=\omega_{r b i} R_{i 1}$

here, $\omega_{r b i}$ are the angular velocities of $\boldsymbol{R}_{i 1}$.

Differentiating both sides of Eq. (23a) with respect to time, it leads to

$\left(v_{r c i} \delta_{c i}+\omega_{r c i} \times r_{c i}\right) \cdot R_{i 2}+r_{c i} \cdot\left(\omega \times R_{i 2}\right)=0$

$\left(\omega_{r c i} \times r_{c i}\right) \cdot \boldsymbol{R}_{i 2}=-r_{c i} \cdot\left(\omega \times \boldsymbol{R}_{i 2}\right)$

Dot-multiplying Eq. (22) with $\boldsymbol{R}_{\mathrm{i} 2}$ at both sides and by means of (23a and $24 b)$, it leads to

$v_{r b i} \delta_{b i} \cdot \boldsymbol{R}_{i 2}+\omega_{r b i}\left(\boldsymbol{R}_{i 1} \times \boldsymbol{r}_{b i}\right) \cdot \boldsymbol{R}_{i 2}-\left(\boldsymbol{R}_{i 2} \times \boldsymbol{r}_{c i}\right) \cdot \omega=\left(\boldsymbol{v}+\omega \times \boldsymbol{e}_{i}\right) \cdot \boldsymbol{R}_{i 2}$ $v_{r b i}\left(\delta_{b i} \cdot \boldsymbol{R}_{i 2}\right)+\omega_{r b i}\left[\left(\boldsymbol{R}_{i 1} \times \boldsymbol{r}_{b i}\right) \cdot \boldsymbol{R}_{i 2}\right]=\left[\begin{array}{ll}\boldsymbol{R}_{i 2}^{T} & {\left[\left(\boldsymbol{e}_{i}-\boldsymbol{r}_{c i}\right) \times \boldsymbol{R}_{i 2}\right]^{T}}\end{array}\right]\left[\begin{array}{c}\boldsymbol{v} \\ \omega\end{array}\right]$

Dot-multiplying Eq.(22) with $\boldsymbol{\delta}_{c i}$ at both sides, it leads to

$v_{r b i} \delta_{b i} \cdot \delta_{c i}+\left(\omega_{r b i} \times r_{b i}\right) \cdot \delta_{c i}+v_{r c i}=\left(v+\ddot{a} \times e_{i}\right) \cdot \delta_{c i}$

Substituting Eq. (23b) into Eq. (26a), it leads to

$v_{r b i} \delta_{b i} \cdot \delta_{c i}+\omega_{r b i}\left(R_{i 1} \times r_{b i}\right) \cdot \delta_{c i}+v_{r c i}=\left(v+\omega \times e_{i}\right) \cdot \delta_{c i}$

$=\left[\begin{array}{ll}\delta_{c i}^{T} & \left(e_{i} \times \delta_{c i}\right)^{T}\end{array}\right]\left[\begin{array}{c}v \\ \omega\end{array}\right]$

The velocity of $b_{\mathrm{i}}$ can be expressed as following

$\dot{b}_{i}=v_{r b i} \delta_{b i}+\omega_{r b i} \times r_{b i}=v_{r b i} \delta_{b i}+\omega_{r b i}\left(R_{i 1} \times r_{b i}\right)$

Since $b_{1} b_{2} b_{3}$ is an equilateral triangle, the dimension constrained equations can be expressed as follows:

$\left(b_{i}-b_{j}\right) \cdot\left(b_{i}-b_{j}\right)=d^{2}, i=1,2,3 ; j=1,2,3 ; i \neq j$

Differentiating both sides of Eq. (28a) with respect to time yields

$\left(\boldsymbol{b}_{\boldsymbol{i}}-\boldsymbol{b}_{\boldsymbol{j}}\right) \cdot\left(\boldsymbol{b}_{\boldsymbol{i}}-\boldsymbol{b}_{\boldsymbol{j}}\right)=0, \boldsymbol{i}=1,2,3 ; \boldsymbol{j}=1,2,3 ; \boldsymbol{i} \neq \boldsymbol{j}$

From Eq. (28b), it leads to

$\left(\dot{\boldsymbol{b}}_{2}-\dot{\boldsymbol{b}}_{1}\right) \cdot\left(\boldsymbol{b}_{2}-\boldsymbol{b}_{1}\right)=0$

$\left(\dot{\boldsymbol{b}}_{3}-\dot{\boldsymbol{b}}_{1}\right) \cdot\left(\boldsymbol{b}_{3}-\boldsymbol{b}_{1}\right)=0$

$\left(\dot{\boldsymbol{b}}_{2}-\dot{\boldsymbol{b}}_{3}\right) \cdot\left(\boldsymbol{b}_{2}-\boldsymbol{b}_{3}\right)=0$

From (25b), it leads to

$\boldsymbol{v}_{r b 1}\left(\delta_{b 1} \cdot \boldsymbol{R}_{12}\right)+\omega_{r b 1}\left[\left(\boldsymbol{R}_{11} \times \boldsymbol{r}_{b 1}\right) \cdot \boldsymbol{R}_{12}\right]=\left[\begin{array}{ll}\boldsymbol{R}_{12}^{T} & {\left[\left(\boldsymbol{e}_{1}-\boldsymbol{r}_{c 1}\right) \times \boldsymbol{R}_{12}\right]^{T}}\end{array}\right]\left[\begin{array}{c}\boldsymbol{v} \\ \omega\end{array}\right]$

$\boldsymbol{v}_{r b 2}\left(\delta_{b 2} \cdot \boldsymbol{R}_{22}\right)+\omega_{r b 2}\left[\left(\boldsymbol{R}_{21} \times \boldsymbol{r}_{b 2}\right) \cdot \boldsymbol{R}_{22}\right]$

$\left.=\left[\begin{array}{ll}\boldsymbol{R}_{22}^{T} & {\left[\left(\boldsymbol{e}_{2}-\boldsymbol{r}_{c 2}\right) \times \boldsymbol{R}_{22}\right.}\end{array}\right]^{T}\right]\left[\begin{array}{c}\boldsymbol{v} \\ \omega\end{array}\right]$

$v_{r b 3}\left(\delta_{b 3} \cdot R_{32}\right)+\omega_{r b 3}\left[\left(R_{31} \times r_{b 3}\right) \cdot R_{32}\right]$

$\left.=\left[\begin{array}{cc}R_{32}^{T} & {\left[\left(e_{3}-r_{c 3}\right) \times R_{32}\right.}\end{array}\right]^{T}\right]\left[\begin{array}{c}v \\ \omega\end{array}\right]$

From Eq. (26b), it leads to

$$
\begin{aligned}
& v_{r b 1} \delta_{b 1} \cdot \delta_{c 1}+\omega_{r b 1}\left(R_{11} \times r_{b 1}\right) \cdot \delta_{c 1}+v_{r c 1} \\
& =\left(v+\omega \times e_{1}\right) \cdot \delta_{c 1}=\left[\begin{array}{ll}
\delta_{c 1}^{T} & \left(e_{1} \times \delta_{c 1}\right)^{T}
\end{array}\right]\left[\begin{array}{c}
v \\
\omega
\end{array}\right] \\
& v_{r b 2} \delta_{b 2} \cdot \delta_{c 2}+\omega_{r b 2}\left(R_{21} \times r_{b 2}\right) \cdot \delta_{c 2}+v_{r c 2} \\
& =\left(v+\omega \times e_{2}\right) \cdot \delta_{c 2}=\left[\begin{array}{ll}
\delta_{c 2}^{T} & \left(e_{2} \times \delta_{c 2}\right)^{T}
\end{array}\right]\left[\begin{array}{c}
v \\
\omega
\end{array}\right] \\
& v_{r b 3} \delta_{b 3} \cdot \delta_{c 3}+\omega_{r b 3}\left(R_{31} \times r_{b 3}\right) \cdot \delta_{c 3}+v_{r c 3} \\
& =\left(v+\omega \times e_{i}\right) \cdot \delta_{c 3}=\left[\begin{array}{ll}
\delta_{c 3}^{T} & \left(e_{3} \times \delta_{c 3}\right)^{T}
\end{array}\right]\left[\begin{array}{c}
v \\
\omega
\end{array}\right]
\end{aligned}
$$

From Eq. (25a), it leads to 
Substituting Eq. (27) into Eqs. (28c-e), yield

$\left[v_{r b 1} \delta_{b 1}+\omega_{r b 1}\left(R_{11} \times r_{b 1}\right)-v_{r b 2} \delta_{b 2}-\omega_{r b 2}\left(R_{21} \times r_{b 2}\right)\right] \cdot\left(b_{1}-b_{2}\right)=0$

$\left[v_{r b 3} \delta_{b 3}+\omega_{r b 3}\left(R_{31} \times r_{b 3}\right)-v_{r b 1} \delta_{b 1}-\omega_{r b 1}\left(R_{11} \times r_{b 1}\right)\right] \cdot\left(b_{3}-b_{1}\right)=0$

$\left[v_{r b 2} \delta_{b 2}+\omega_{r b 2}\left(R_{21} \times r_{b 2}\right)-v_{r b 3} \delta_{b 3}-\omega_{r b 3}\left(R_{31} \times r_{b 3}\right)\right] \cdot\left(b_{2}-b_{3}\right)=0$

Where

$$
\delta_{m 1}=\frac{\boldsymbol{b}_{1}-\boldsymbol{b}_{2}}{\left|\boldsymbol{b}_{1}-\boldsymbol{b}_{2}\right|}, \delta_{m 2}=\frac{\boldsymbol{b}_{3}-\boldsymbol{b}_{1}}{\left|\boldsymbol{b}_{3}-\boldsymbol{b}_{1}\right|}, \delta_{m 3}=\frac{\boldsymbol{b}_{2}-\boldsymbol{b}_{3}}{\left|\boldsymbol{b}_{2}-\boldsymbol{b}_{3}\right|}
$$

Eqs. (29a-i) can be expressed in matrix form as

$\mathrm{J}_{\alpha} v_{m}=\mathrm{J}_{\lambda} v_{s}$



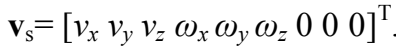

$$
\begin{aligned}
& \mathbf{J}_{\alpha}=\left[\begin{array}{ccccccccc}
\delta_{b 1} \cdot R_{12} & 0 & 0 & 0 & 0 & 0 & \left(R_{11} \times r_{b 1}\right) \cdot R_{12} & 0 & 0 \\
0 & \delta_{b 2} \cdot R_{22} & 0 & 0 & 0 & 0 & 0 & \left(R_{21} \times r_{b 2}\right) \cdot R_{22} & 0 \\
0 & 0 & \delta_{b 3} \cdot R_{32} & 0 & 0 & 0 & 0 & 0 & \left(R_{31} \times r_{b 3}\right) \cdot R_{32} \\
\delta_{b 1} \cdot \delta_{c 1} & 0 & 0 & 1 & 0 & 0 & \left(R_{11} \times r_{b 1}\right) \cdot \delta_{c 1} & 0 & 0 \\
0 & \delta_{b 2} \cdot \delta_{c 2} & 0 & 0 & 1 & 0 & 0 & \left(R_{21} \times r_{b 2}\right) \cdot \delta_{c 2} & 0 \\
0 & 0 & \delta_{b 3} \cdot \delta_{c 3} & 0 & 0 & 1 & 0 & 0 & \left(R_{31} \times r_{b 3}\right) \cdot \delta_{c 3} \\
\delta_{m 1} \cdot \delta_{b 1} & -\delta_{m 1} \cdot \delta_{b 2} & 0 & 0 & 0 & 0 & \delta_{m 1} \cdot\left(R_{11} \times r_{b 1}\right) & -\delta_{m 1} \cdot\left(R_{21} \times r_{b 2}\right) & 0 \\
-\delta_{m 2} \cdot \delta_{b 1} & 0 & \delta_{m 2} \cdot \delta_{b 3} & 0 & 0 & 0 & -\delta_{m 2} \cdot\left(R_{11} \times r_{b 1}\right) & 0 & \delta_{m 2} \cdot\left(R_{31} \times r_{b 3}\right) \\
0 & \delta_{m 3} \cdot \delta_{b 2} & -\delta_{m 3} \cdot \delta_{b 3} & 0 & 0 & 0 & 0 & \delta_{m 3} \cdot\left(R_{21} \times r_{b 2}\right) & -\delta_{m 3} \cdot\left(R_{31} \times r_{b 3}\right)
\end{array}\right] \\
& \mathbf{J}_{\lambda}=\left[\begin{array}{ccc}
R_{12}^{T} & {\left[\left(e_{1}-r_{b 1}\right) \times R_{12}\right]^{T}} & 0_{1 \times 3} \\
R_{22}^{T} & {\left[\left(e_{2}-r_{b 2}\right) \times R_{22}\right]^{T}} & 0_{1 \times 3} \\
R_{32}^{T} & {\left[\left(e_{3}-r_{b 3}\right) \times R_{32}\right]^{T}} & 0_{1 \times 3} \\
\delta_{c 1}^{T} & \left(e_{1} \times \delta_{c 1}\right)^{T} & 0_{1 \times 3} \\
\delta_{c 2}^{T} & \left(e_{2} \times \delta_{c 2}\right)^{T} & 0_{1 \times 3} \\
\delta_{c 3}^{T} & \left(e_{3} \times \delta_{c 3}\right)^{T} & 0_{1 \times 3} \\
0_{1 \times 3} & 0_{1 \times 3} & 0_{1 \times 3} \\
0_{1 \times 3} & 0_{1 \times 3} & 0_{1 \times 3} \\
0_{1 \times 3} & 0_{1 \times 3} & 0_{1 \times 3}
\end{array}\right],
\end{aligned}
$$

From Eq. (30a), it leads to

$v_{m}=\mathbf{J} v_{s}=\mathbf{J}_{\alpha}^{-1} \mathbf{J}_{\lambda} v_{s}$

Eq. (30b) has nine rows and nine columns. From the first six rows and the first six columns, it leads to

$v_{r}=\mathbf{J}_{f}\left[\begin{array}{c}v \\ \omega\end{array}\right]$

here, $\boldsymbol{v}_{r}=\left[\begin{array}{lllllll}v_{r b 1} & v_{r b 2} & v_{r b 3} & v_{r c 1} & v_{r c 2} & v_{r c 3}\end{array}\right]^{T}, \mathbf{J}_{\mathrm{f}}$ is the inverse Jacobian matrix.

\section{DRIVING FORCES ANALYSIS}

Let $f_{r b i}$ and $f_{r c i}(i=1,2,3)$ be the active forces along limbs $r_{b i}$ and $r_{c i}(i=1,2,3)$, respectively. Let $\boldsymbol{F}$ and $\boldsymbol{T}$ be a central force and a central torque applied onto $m$ at $o$. Based on the principle of virtual work, it leads to

$$
\boldsymbol{f}_{r}^{T} \boldsymbol{v}_{r}+\left[\begin{array}{ll}
\boldsymbol{F}^{T} & \boldsymbol{T}^{T}
\end{array}\right]\left[\begin{array}{c}
\boldsymbol{v} \\
\boldsymbol{\omega}
\end{array}\right]=0
$$

From Eq. (32), it leads to
$\boldsymbol{f}_{r}=-\left(\mathbf{J}_{f}^{-1}\right)^{T}\left[\begin{array}{l}\boldsymbol{F} \\ \boldsymbol{T}\end{array}\right]$

Where, $\boldsymbol{f}_{\mathrm{r}}=\left[f_{\mathrm{rb} 1} f_{\mathrm{rb} 2} f_{\mathrm{rb} 3} f_{\mathrm{rc} 1} f_{\mathrm{rc} 2} f_{\mathrm{rc} 3}\right]^{\mathrm{T}}, \boldsymbol{F}=\left[F_{\mathrm{x}} F_{\mathrm{y}} F_{\mathrm{z}}\right]^{\mathrm{T}}, \boldsymbol{T}=\left[T_{\mathrm{x}} T_{\mathrm{y}}\right.$ $\left.T_{\mathrm{z}}\right]^{\mathrm{T}}$.

\section{NUMERICAL EXAMPLE}

Set $E=120 / q, C=60 / q, d=80 \mathrm{~cm} . \boldsymbol{F}=[-20,-30,-30]^{\mathrm{T}} \mathrm{kN}$, $T=[-30-30,100] \mathrm{kNcm}$. The pose parameters of the moving platform are given as $X_{0}=68.89, Y_{0}=49.63, Z_{0}=225.3 \mathrm{~cm}, \alpha=$ $-11.08, \beta=32.97, \gamma=-12.88^{\circ}$. The velocity parameters of moving platform are given as $v_{x}=1, v_{y}=2, v_{z}=3 \mathrm{~cm} / \mathrm{s}, \omega_{x}=1$, $\omega_{y}=2, \omega_{z}=3 \%$, the position vectors of $b_{i}(i=1,2,3)$ are solved as Table 1, 2 and $\mathbf{3}$.

Table 1. The Coordinates of $b_{1}$

\begin{tabular}{|c|c|c|c|c|}
\hline $\boldsymbol{b}_{\mathbf{1}}$ & $\mathbf{1}$ & $\mathbf{2}$ & $\mathbf{3}$ & $\mathbf{4}$ \\
\hline \hline$X(\mathrm{~cm})$ & 50.667 & 45.141 & 39.998 & 21.167 \\
\hline$Y(\mathrm{~cm})$ & -29.253 & -26.06 & -23.093 & -12.22 \\
\hline$Z(\mathrm{~cm})$ & 115.64 & 116.73 & 117.74 & 121.44 \\
\hline & 5 & 6 & 7 & 8 \\
\hline$X(\mathrm{~cm})$ & $-90.26+167.89 \mathrm{i}$ & -51.398 & -40.555 & $-90.26-167.89 \mathrm{i}$ \\
\hline$Y(\mathrm{~cm})$ & $52.111-96.931 \mathrm{i}$ & 29.674 & 23.415 & $52.111+96.931 \mathrm{i}$ \\
\hline$Z(\mathrm{~cm})$ & $143.37-33.038 \mathrm{i}$ & 135.72 & 133.59 & $143.37+33.038 \mathrm{i}$ \\
\hline
\end{tabular}

Table 2. The Coordinates of $b_{2}$

\begin{tabular}{|c|c|c|c|c|}
\hline $\boldsymbol{b}_{2}(\mathbf{c m})$ & $\mathbf{1}$ & $\mathbf{2}$ & $\mathbf{3}$ & $\mathbf{4}$ \\
\hline \hline$X$ & 0 & 0 & 0 & 0 \\
\hline$Y$ & 32.471 & 39.947 & 46.19 & 64.583 \\
\hline$Z$ & 120.44 & 118.98 & 117.75 & 114.15 \\
\hline $\boldsymbol{b}_{2}(\mathrm{~cm})$ & 5 & 6 & 7 & 8 \\
\hline$X$ & 0 & 0 & 0 & 0 \\
\hline$Y$ & $-138.95-172.52 \mathrm{i}$ & -31.569 & -45.511 & $-138.95+172.52 \mathrm{i}$ \\
\hline$Z$ & $154.01+33.785 \mathrm{i}$ & 132.98 & 135.71 & $154.01-33.785 \mathrm{i}$ \\
\hline
\end{tabular}

Table 3. The Coordinates of $b_{3}$

\begin{tabular}{|c|c|c|c|c|}
\hline $\boldsymbol{b}_{\mathbf{3}}(\mathbf{c m})$ & $\mathbf{1}$ & $\mathbf{2}$ & $\mathbf{3}$ & $\mathbf{4}$ \\
\hline \hline$X$ & 64.528 & 65.347 & -37.528 & 63.356 \\
\hline$Y$ & 37.255 & 37.728 & -21.667 & 36.579 \\
\hline$Z$ & 73.394 & 72.88 & 137.43 & 74.13 \\
\hline $\boldsymbol{b}_{3}$ & 5 & 6 & 7 & 8 \\
\hline$X$ & $-184.96+13.944 \mathrm{i}$ & -74.269 & 29.45 & $-184.96-13.944 \mathrm{i}$ \\
\hline$Y$ & $-106.79+8.0503 \mathrm{i}$ & -42.879 & 17.003 & $-106.79-8.0503 \mathrm{i}$ \\
\hline$Z$ & $229.93+8.75 \mathrm{i}$ & 160.48 & 95.40 & $229.93+8.75 \mathrm{i}$ \\
\hline
\end{tabular}


Table 4. Inverse Solutions of 3RPS-3SPR PM

\begin{tabular}{|c|c|c|c|c|c|c|}
\hline $\mathbf{( c m )}$ & $\mathbf{1}$ & $\mathbf{2}$ & $\mathbf{3}$ & $\mathbf{4}$ & $\mathbf{5}$ & $\mathbf{6}$ \\
\hline \hline$r_{b 1}$ & 116.1401 & 117.9807 & 119.9824 & 129.4582 & 186.9944 & 176.9975 \\
\hline$r_{b 2}$ & 125.9397 & 122.5390 & 119.9962 & 114.2482 & 166.8976 & 177.7500 \\
\hline$r_{b 3}$ & 161.4403 & 162.0518 & 139.8558 & 160.5745 & 161.3236 & 140.6064 \\
\hline$r_{c 1}$ & 125.1317 & 124.9137 & 125.0120 & 127.8178 & 167.8407 & 159.6376 \\
\hline$r_{c 2}$ & 122.7075 & 121.0418 & 120.0042 & 118.8893 & 152.6122 & 161.9011 \\
\hline$r_{c 3}$ & 175.3520 & 175.9541 & 150.1267 & 174.4991 & 168.4908 & 154.4953 \\
\hline
\end{tabular}

Table 5. Velocity and Statics at the Reasonable Pose

\begin{tabular}{|c|c|c|c|c|c|}
\hline $\boldsymbol{v}_{r b 1}(\mathbf{c m} / \mathbf{s})$ & $\boldsymbol{v}_{r b 2}(\mathbf{c m} / \mathbf{s})$ & $\boldsymbol{v}_{r b 3}(\mathbf{c m} / \mathbf{s})$ & $\boldsymbol{v}_{r c 1}(\mathbf{c m} / \mathbf{s})$ & $\boldsymbol{v}_{r c 2}(\mathbf{c m} / \mathbf{s})$ & $\boldsymbol{v}_{r c 3}(\mathbf{c m} / \mathbf{s})$ \\
\hline \hline-3.9609 & 11.9576 & -31.7202 & 9.1684 & -9.9490 & 35.6654 \\
\hline$f_{r b 1}(\mathrm{kN})$ & $f_{r b 2}(\mathrm{kN})$ & $f_{r b 3}(\mathrm{kN})$ & $f_{r c 1}(\mathrm{kN})$ & $f_{r c 2}(\mathrm{kN})$ & $f_{r c 3}(\mathrm{kN})$ \\
\hline-48.9508 & -104.8954 & 78.6797 & -22.1896 & -81.8418 & 79.9695 \\
\hline
\end{tabular}

From the results, it can be seen that there are eight solutions of the position vectors of $b_{i}(i=1,2,3)$, this means that the inverse displacement of the 3 RPS-3SPR PM has eight solutions. However, not all of them are real solutions. The real solutions are used to solve inverse displacement as follows:

By using the CAD variation geometry approach proposed in reference [9], a simulation mechanism of this PM can be created and the forward displacement solutions can be derived subsequently. By comparing, it is known that the simulation solution coincides with the third solution of analytical result in Table 4 . Then the velocity and statics are calculated at this pose as following (See Table 5)

\section{WORKSPACE ANALYSIS}

The workspace of this S-PM can be created using CAD variation geometry approach [9]. When given the maximum

Table 6. The Construction Processes of Sub-Workspace

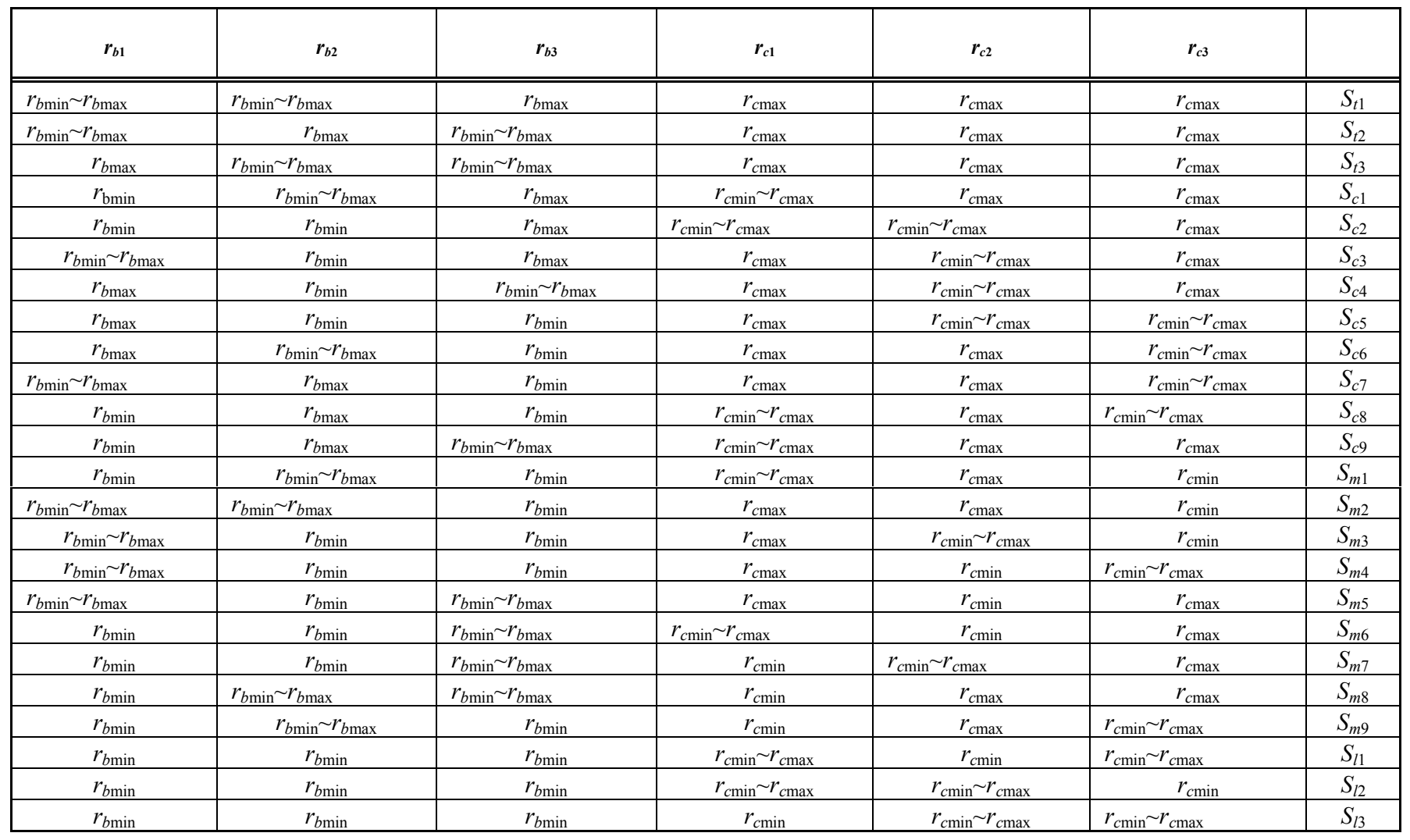



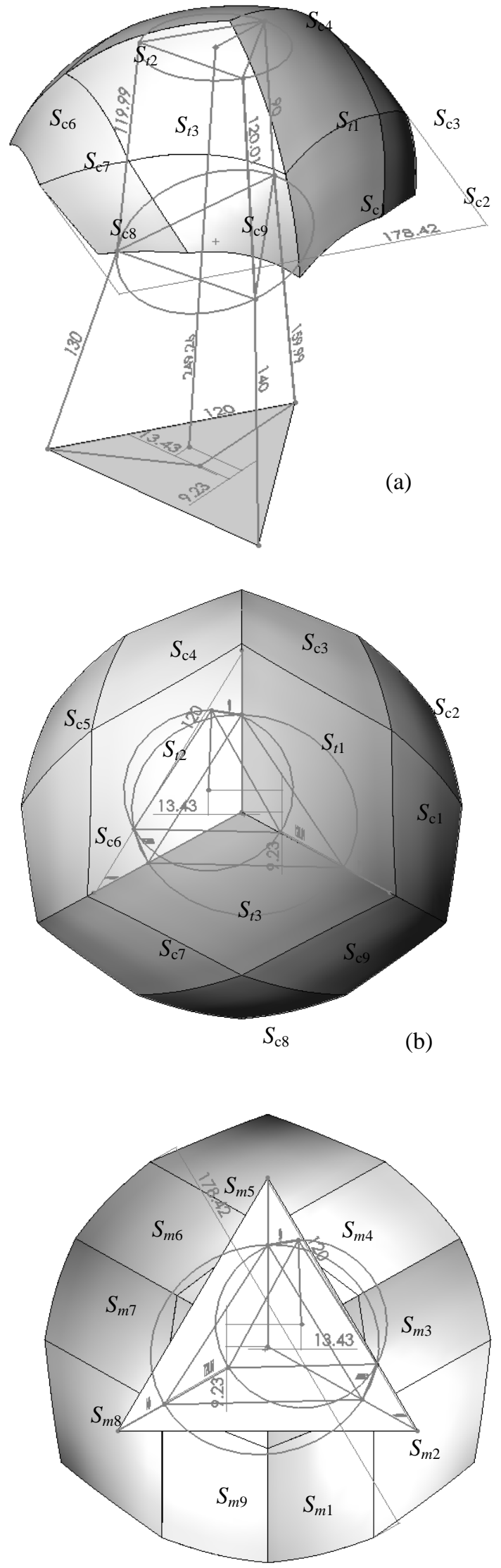

(c)

Fig. (2). A reachable workspace $W$ of the 3RPS-3SPR S-PM. (a) the isometric view, (b) the top view, (c) the upper view. extension $r_{b \max }$, the minim extension $r_{b \operatorname{mim}}$ and the increment $\delta r_{b}$ of active legs $r_{b i}(i=1,2,3)$, the maximum extension $r_{c \max }$, the minim extension $r_{c \mathrm{cmim}}$ and the increment $\delta r_{c}$ of active legs $r_{c i}(i=1,2,3)$, the reachable workspace $W$ of the 3RPS3SPR S-PM can be constructed by its simulation mechanism.

When four of $r_{b i}$ and $r_{c i}(i=1,2,3)$ reach their limited values of (rbmin, $\left.r_{b \max }, r_{c \min }, r_{c \max }\right)$, varying the remaining two of $r_{b i}$ and $r_{c i}(i=1,2,3)$ from $r_{b \text { min }}$ to $r_{b \max }$ and from $r_{c \text { min }}$ to $r_{c \max }$, respectively, each sub-workspace can be constructed.

The construction processes are described as follows:

Step 1. Set $r_{b 3}=r_{b \max }, r_{c 1}=r_{c 2}=r_{c 3}=r_{c \max }$.

Step 2. Set $r_{b 1}=r_{b \min }+(j-1) \delta r_{b}\left(j=1, \ldots, n_{1}\right)$,

where $n_{1}=\left(r_{b \max }-r_{b \min }\right) / \delta r_{b}$.

Step 3. Set $j=1$ and increase $r_{b 2}$ by $\delta r_{b}$ at each increment from $r_{b \min }$ to $r_{b \max }$. Solve the position components $\left(X_{o} Y_{o} Z_{o}\right)$ by using CAD software. Then, a spatial curve $c_{1}$ is formed from the solutions of $\left(X_{o} Y_{o} Z_{o}\right)$ of $n_{1}$ points.

Step 4. Repeat the steps 2 , except that set $\mathrm{j}=2, \ldots, \mathrm{n} 1$, thus other cj can be constructed. Construct the $n_{1}$ spatial curves $c_{j}$ $\left(j=1, \ldots, n_{1}\right)$ by the loft command. Then the surface $S_{t 1}$ can be obtained.

Step 5. Repeat the steps 1-4, except that set $r_{b i}$ and $r_{c i}$ verifying versus Table $\mathbf{6}$, the other sub-workspace can be obtained.

The workspace of 3RPS-3SPR S-PM is constructed as shown in Fig. (2).

\section{CONCLUSIONS}

A novel 3RPS-3SPR serial-parallel manipulator (S-PM) is constructed by connecting a 3RPS parallel manipulator (PM) with a 3SPR PM in series. Some formulae are derived for solving the inverse displacement, velocity, and statics models of this S-PM.

It is known from the analytic solutions that this S-PM has eight inverse solutions. A reasonable solution can be obtained by comparing with the simulation solutions. The inverse velocity and the active forces are computed at this reasonable pose.

A $9 \times 9$ Jacobian matrix is derived from the geometrical constraint and the dimension constraint equations. A $6 \times 6$ Jacobian matrix is derived for solving inverse velocity by taking the first six rows and the first six arranges out of the $9 \times 9$ Jacobian matrix.

The active forces are derived based on the principle of virtual work. Its workspace is constructed by CAD variation geometry approach, this novel S-PM has a large workspace.

This S-PM has some potential applications for the serialparallel machine tools, the sensors, the surgical manipulators and the satellite surveillance platforms. The method for solving the inverse kinematics and workspace can also be used for other S-PMs.

\section{CONFLICT OF INTEREST}

None declared. 


\section{ACKNOWLEDGEMENTS}

The authors would like to acknowledge Project (51175447) supported by the National Natural Sciences Foundation of China (NSFC), the Key planned project of Hebei application foundation (11962127D) and the research and development project of science and technology of Qinhuangdao city (201101A140).

\section{REFERENCES}

[1] J.-P. Merlet, Parallel robots. London: Kluwer Academic Publishers, 2000.

[2] H. Zhen, and L. Qinchuan, "Type synthesis of symmetrical lowermobility parallel mechanisms using constraint-synthesis method", Int. J. Robot Res., vol. 22(1), pp. 59-79, 2003.

[3] G. Feng, L. Weimin, and Z. Xianchao, "New kinematic structures for 2-, 3-, 4-, and 5-dof PM designs", Mech. Mach. Theory, vol. 37(11), pp. 1395-1411, 2002.

[4] X. W. Kong, and C. M. Gosselin, "Type synthesis of 3-DOF spherical PMs based on screw theory”, ASME J. Mach. Des., vol. 126, pp. 101-108, 2004.

[5] S. Joshi, and L. W. Tsai, "Jacobian analysis of limited-DOF PMs", ASME J. Mech. Des., vol. 124(2), pp. 254-258, 2002.

[6] J. Gallardo, H. Orozco, and J. Rico, "Kinematics of 3-RPS parallel manipulators by means of screw theory", Int. J. Adv. Manuf. Tech., vol. 36(5-6), pp. 598-605, 2008 .

[7] Y. $\mathrm{Lu}$, and B. Hu, "A unified approach to solving driving forces in spatial PMs with less than 6-DOF", Trans. ASME J. Mech. Des., vol. 129 (11), pp. 1153-1160, 2007.

[8] K. T. Tanio "Kinematics of a hybrid (parallel-serial) robot manipulator", Mech. Mach. Theory, 35(9), pp. 1183-1196, 2000.

[9] Y. Lu, and B. Hu, "Solving driving forces of 2(3-SPR) serial-PM by CAD variation geometry approach", Trans. ASME J. Mech. Des., vol. 128(6), pp. 1349-1351, 2006.
[10] Y. Lu, B. Hu and T. Sun, "Analyses of velocity, acceleration, statics, and workspace of a 2(3-SPR) serial-parallel manipulator", Robotica, vol. 27(4), pp. 529-538, 2009.

[11] J. Gallardo-Alvarado, C. Aguilar-Najera, L. Casique-Rosas, J. Ricomartinez, and M. Islam, "Kinematics and dynamics of 2(3RPS) manipulators by means of screw theory and the principle of virtual work", Mech. Mach. Theory, vol. 43(10), pp. 1281-1294, 2008

[12] X. Z. Zheng, H. Z. Bin, and Y. G. Luo, "Kinematic analysis of ahybrid serial-parallel manipulator", Int. J. Adv. Manuf. Tech., vol. 23, pp. 925-930, 2004.

[13] I. Ouarda, and K. Wisama, "Inverse and direct dynamic models of hybrid robots", Mech. Mach. Theory, vol. 45(4), pp. 627-640, 2010.

[14] A. A. Ramadan, K. Inoue, T. Arai, and T. Takubo, "New architecture of a hybrid two-fingered micro-nano manipulator hand: optimization and design", Adv. Robot., vol. 22, pp. 235-60, 2008 .

[15] H. Liu, T. Huang, J. Mei, X. Zhao, and H. Derek, "Kinematic design of a 5-DOF hybrid robot with large workspace/limb-stroke ratio. ASME J. Mech. Des., vol. 129(5), pp. 530-537, 2007.

[16] Z. Huang, J. Wang, and Y. F. Fang, "Analysis of instantaneous motions of deficient-rank 3-RPS PMs" Mech. Mach. Theory, vol. 37(2), pp. 229-240, 2002.

[17] Liu C. H., and Cheng S. C., "Direct singular positions of 3RPS PMs”, ASME J. Mech. Des., vol. 126(10), pp. 1006-1016, 2004.

[18] N. M. Rao, and K. M. Rao, "Multi-position dimensional synthesis of a spatial 3-RPS PM", ASME J. Mech. Des., vol. 128(7), pp. 815819, 2006

[19] Y. Lu, and B. Hu, "Unification and simplification of velocity/ acceleration of limited-dof parallel manipulators with linear active legs", Mech. Mach. Theory, vol. 43(9), pp. 1112-1128, 2008.

[20] S.-K. Song, D. S. Kwon, and W. S. Kim, "Spherical joint for coupling three or more links together at one point", US Pat., $6568871,2003$.

(C) Hu et al.; Licensee Bentham Open.

This is an open access article licensed under the terms of the Creative Commons Attribution Non-Commercial License (http://creativecommons.org/licenses/by$\mathrm{nc} / 3.0 /$ ), which permits unrestricted, non-commercial use, distribution and reproduction in any medium, provided the work is properly cited. 